\title{
EFECTO TÓXICO DEL SAÚCO, Sambucus peruviana (CAPRIFOLIACEAE), EN Daphnia magna, Sitophilus zeamais Y Copidosoma koehleri EN PERÚ
}

\author{
TOXIC EFFECT OF PERUVIAN ELDERBERRY, Sambucus peruviana \\ (CAPRIFOLIACEAE), ON Daphnia magna, Sitophilus zeamais, AND Copidosoma \\ koehleri IN PERU
}

\author{
Amid Román-Farje ${ }^{1}$, José Iannacone ${ }^{1,2^{*}}$, y Lorena Alvariño ${ }^{1}$ \\ ${ }^{1}$ Laboratorio de Ecología y Biodiversidad Animal (LEBA), Facultad de Ciencias Naturales y Matemática \\ (FCNNM), Universidad Nacional Federico Villarreal (UNFV), El Agustino, Lima, Perú. \\ ${ }^{2}$ Facultad de Ciencias Biológicas, Universidad Ricardo Palma (URP), Santiago de Surco, Lima, Perú \\ * Autor para correspondencia E-mail: joseiannacone@gmail.com
}

\section{RESUMEN}

El uso de extractos de plantas con propiedades insecticidas es una alternativa al uso de agroquímicos sintéticos. Se evaluó el efecto tóxico del saúco, Sambucus peruviana Kuhnt (Caprifoliaceae), en el crustáceo pulga de agua, Daphnia magna Straus (Cladocera, Daphniidae) y la microavispa Copidosoma koehleri Blanchard (Hym.: Encyrtidae). La hoja, corteza y raíz de saúco se evaluaron en maceración en agua, cocción, infusión y maceración en alcohol etílico. También se evaluó el efecto del polvo de corteza en el gorgojo del maíz, Sitophilus zeamais (Motschulsky) (Col.: Curculionidae). Los mayores efectos de toxicidad para D. magna se produjeron con la infusión de hojas. Los adultos de $C$. koehleri fueron más sensibles a la infusión de raíces. La emergencia de las microavispas adultas disminuyó con la cocción de hojas. Los estados larvarios y pupas no emergidos fueron afectados por la cocción y maceración de hojas. También se observó repelencia, de mayor nivel con la maceración en alcohol etílico de hojas y raíces. El polvo de corteza causó mortalidad en S. zeamais. También hubo efectos de la maceración, cocción e infusión de la corteza en el porcentaje de granos dañados, los que perdieron < del $3 \%$ de su peso. El efecto insecticida y repelente del polvo y la cocción de la corteza de S. peruviana es importante por su efecto en S. zeamais, pero no así las hojas y raíces, por sus efectos negativos en el ambiente acuático de $D$. magna y en el control biológico por $C$. koehleri.

Palabras clave: Grano almacenado, efecto insecticida, efecto repelente, insecticida botánico.

\section{ABSTRACT}

Some extracts of plants with insecticidal properties are used on pest control as an alternative to synthetic agrochemicals. The objective of this study was to evaluate the toxic effect of Peruvian elderberry, Sambucus peruviana Kuhnt (Caprifoliaceae), on the water flea Daphnia magna Straus, the maize weevil Sitophilus zeamais (Motschulsky) (Col.: Curculionidae), and the microhymenopteran Copidosoma koehleri Blanchard (Hym.: Encyrtidae). Plant leaves, bark, and roots were treated by maceration in water, boiling, infusion and maceration in ethyl alcohol. The insecticidal activity of root and bark powders against Sitophilus zeamais was also evaluated. The highest repellent activity against $D$. magna was observed with the use of leaf infusion. Adults of $C$. koehleri were more sensitive to root infusion. Adult emergence of this parasitoid decreased with leaves macerated in water. Larvae and pupae did not emerge with leaf treatments by maceration and boiling. Higher levels of repellency were observed with leaves and roots treated by maceration in ethyl alcohol. 
Bark powder caused mortality of $S$. zeamais. Bark treated by maceration, boiling, and as an infusion also had effects on the percentage of damaged grains, resulting in a $<3 \%$ weight loss. It can be concluded that unlike $S$. peruviana leaves and roots, both powder and boiled bark have insecticidal and repellent effects against $S$. zeamais.

Key words: Insecticidal effect, plant insecticide, repellent effect, stored grain.

\section{INTRODUCCIÓN}

Algunas plantas con propiedades insecticidas se utilizan en el control de plagas agrícolas como alternativa al uso de agroquímicos, los que tienen consecuencias negativas en el agroecosistema y las personas, aumentan el costo de producción, y pueden causar desequilibrios ecológicos al afectar a los enemigos naturales de las plagas y especialmente los ecosistemas acuáticos (Iannacone et al., 2007; Akob and Ewete, 2007; Iannacone y Alvariño, 2010).

Los plaguicidas botánicos se pueden utilizar en conjunto con la fauna benéfica para regular las plagas. Las plantas con estas propiedades deben fomentarse ya que causan un menor impacto ecotoxicológico acuático y terrestre, por lo que el conocimiento de los efectos tóxicos y repelentes de los extractos botánicos sobre organismos acuáticos y la fauna benéfica es indispensable para su utilización (Iannacone et al., 2015). Por otra parte, la demanda creciente de alimentos orgánicos y el uso de los insecticidas locales de origen botánico ha aumentado globalmente (Juárez-Flores et al., 2010; Nuñez et al., 2010).

Saúco Sambucus peruviana HBK (Caprifoliaceae) es una planta andina utilizada por su sombra y valor ornamental, distribuida desde Costa Rica hasta el cono sur, entre 2000 y 4000 msnm. Sus frutos sirven de alimento, su follaje tiene propiedades afrodísiacas y medicinales para el hombre, y su madera es de alta calidad. Los extractos de flores y hojas presentan actividad antimicrobiana (Díaz et al., 2004; Cruz et al., 2011). Los principios activos son taninos, flavonoides, saponinas, esteroides, alcaloides, mucílagos, catequinas, quinonas, lactosas, cumarinas y antocianinas (Alvarado-Chávez, 2007; Ruiz et al., 2013; Natividad-Bardales et al., 2014; Torres et al., 2015).

El crustáceo Daphnia magna Straus se utiliza en ensayos para evaluar la toxicidad de xenobióticos (Díaz et al., 2004; APHA, 2012). Este organismo es fácil de desarrollar en laboratorio, tiene un ciclo vital corto, representa al zooplancton en estudios ecotoxicológicos y permite determinar el riesgo ambiental acuático (Iannacone et al., 2007; Iannacone et al., 2014).

El gorgojo del maíz, Sitophilus zeamais (Motschulsky) (Coleoptera: Curculionidae) es una plaga clave del maíz en almacenamiento, donde causa daño significativo si no se le controla (Rodríguez-Cobos y Iannacone 2012). Se ha evaluado el efecto insecticida de diversas plantas promisorias para el control de este gorgojo (Iannacone y Quispe, 2004; Iannacone et al., 2004; Tapondjou et al., 2005; Nuñez et al., 2010; Fedaku et al., 2012).

Copidosoma koehleri Blanchard es un parasitoide nativo de Sudamérica con poliembrionía, que actúa especialmente sobre los huevecillos del complejo de polillas de la papa (Keasar y Steinberg, 2008; Segoli et al., 2009). Sobre esta microavispa se ha evaluado el efecto tóxico de diversos plaguicidas sintéticos y extractos botánicos (Iannacone y Lamas, 2003; Iannacone et al., 2007).

En este trabajo se evaluó el efecto tóxico de preparados en agua y alcohol etílico de hojas, raíces y corteza de S. peruviana en D. magna, S. zeamais y $C$. koehleri.

\section{MATERIALES Y MÉTODOS}

Los bioensayos se realizaron en el Laboratorio de Ecología y Biodiversidad Animal (LEBA) de la Facultad de Ciencias Naturales y Matemática de la Universidad Nacional Federico Villarreal (UNFV), El Agustino, Lima, Perú.

\section{Material biológico}

Sambucus peruviana. La selección de plantas, preparación de las muestras y obtención del polvo seco y extractos botánicos crudos siguieron el protocolo de Iannacone et al. (2014). Las plantas se colectaron en el Distrito de Viques (12 ${ }^{\circ}{ }^{\prime}$ S, $75^{\circ} 13^{\prime}$ W), Provincia de Huancayo Junín, Perú. Para los tratamientos se utilizaron hojas, corteza y raíces. Para la preparación en seco, las partes de la planta se secaron $96 \mathrm{~h}$ en estufa a $37^{\circ} \mathrm{C}$. Las hojas secas se pulverizaron en un mortero (Haldenwanger ${ }^{\circledR}$ ), y las raíces y corteza en un molinillo. Los polvos se pasaron por dos tamices, de 400 y $500 \mu \mathrm{m}$, y se sellaron en frascos de vidrio ámbar para evitar la fotólisis, se rotularon y se mantuvieron en refrigeración hasta los bioensayos (Iannacone et al., 2014).

Los polvos se utilizaron para preparar extractos acuosos de los compuestos hidrosolubles con agua destilada por al menos $7 \mathrm{~d}$ con aireación, todos al 20\% (concentración referencial máxima), por maceración en agua, cocción e infusión, y un extracto con alcohol etílico. Luego se filtraron con papel Whatman ${ }^{\circledR}$ № 5 . En los bioensayos se utili- 
zaron sólo extractos en agua de hasta $72 \mathrm{~h}$ desde la preparación, para evitar pérdidas de calidad por microorganismos fúngicos (Iannacone et al., 2014). Todos los tratamientos se hicieron a la temperatura del laboratorio.

Daphnia magna. Los ejemplares se obtuvieron de un acuario en el distrito de San Isidro, Lima, Perú, que se llevaron al laboratorio y pusieron en recipientes de $2 \mathrm{~L}$ a $\sim 22 \pm 2^{\circ} \mathrm{C}$, según el procedimiento de Díaz et al. (2004) mientras duraron los bioensayos. Solo se utilizaron individuos neonatos $(<24 \mathrm{~h})$ (Iannacone et al., 2014).

Sitophilus zeamais. La crianza de laboratorio se inició a partir de una población de adultos que se obtuvo de maíz desde un mercado del distrito de Surquillo, Lima, Perú, los que se llevaron a envases plásticos de $1 \mathrm{~L}$, de $11,5 \mathrm{~cm}$ de alto, $10 \mathrm{~cm}$ de diámetro de base y $12,5 \mathrm{~cm}$ de diámetro superior (Rodríguez-Cobos y Iannacone, 2012), que se mantuvieron en cámara a $\sim 22 \pm 2^{\circ} \mathrm{C}$ para su reproducción y obtener los adultos para los bioensayos. Los experimentos de 3-7 d se hicieron con adultos no sexados escogidos al azar desde la crianza (lannacone et al., 2004; Tapondjou et al., 2005).

Copidosoma koehleri. Los ejemplares se obtuvieron del CIP (Centro Internacional de la Papa), Lima, Perú, y se criaron en laboratorio sobre larvas de Phthorimaea operculella Zeller a $22 \pm 2^{\circ} \mathrm{C}$ y fotoperíodo 12:12 (Iannacone et al., 2007).

Bioensayos con polvos secos. De los envases de crianza se escogieron al azar adultos no sexados de $S$. zeamais, sobre $10 \mathrm{~g}$ de maíz en envases plásticos de 2,5 cm de alto, 2,3 cm de ancho y $3,3 \mathrm{~cm}$ de largo), con cuatro repeticiones del tratamiento a la mayor concentración $\left(1,6 \mathrm{~g} 10 \mathrm{~g}\right.$ maíz $\left.^{-1}\right)$ y un control con agua destilada. Se agregaron los polvos secos en cada repetición, y se pusieron 10 gorgojos en cada envase. Las evaluaciones se hicieron cada $24 \mathrm{~h}$ durante $5 \mathrm{~d}(=120 \mathrm{~h})$, para determinar el efecto tóxico de los polvos secos sobre el gorgojo (Iannacone et al., 2004). Se calculó el porcentaje de pérdida de peso $(\mathrm{Pp})$ de granos dañados $(\mathrm{Ngd})$ con respecto al total de granos (Ntg) por envase, mediante la fórmula $\mathrm{Pp}=(\mathrm{Ngd} / \mathrm{Ntg})^{*} 100^{*} \mathrm{C}$, donde C es una constante con valor 0,125 si el maíz está almacenado como grano suelto o mazorca sin chalas, como en los bioensayos, o 0,222 si el maíz está almacenado como mazorca con ellas. El bioensayo de toxicidad de polvos secos no se hizo sobre $D$. magna ni C. koehleri.

Maceración. Los extractos en agua destilada se prepararon a $20 \mathrm{~g}$ por $100 \mathrm{~mL}$ agua $(\mathrm{pH}=7,2 ; \mathrm{CE}=$ $0,86 \mathrm{dS} \mathrm{cm}^{-1}$; Calcio $=6,17 \mathrm{me} \mathrm{L}^{-1}$; Magnesio $=1,26$ me L ${ }^{-1}$; Potasio $=0,10$ me L $^{-1}$; Sodio $=1,58$ me L $^{-1}$; Nitratos $=0,10 \mathrm{me} \mathrm{L}^{-1}$; Carbonatos $=0,00 \mathrm{me} \mathrm{L}^{-1}$; Bicarbonatos $=3,55 \mathrm{me} \mathrm{L}^{-1}$; Sulfatos $=2,30 \mathrm{me} \mathrm{L}^{-1}$; Cloruros $=2,40 \mathrm{me} \mathrm{L}^{-1}$; RAS $=0,81 \mathrm{me} \mathrm{L}^{-1}$; Boro $=$ $0,50 \mathrm{me} \mathrm{L}^{-1}$; Dureza $=362 \mathrm{mg} \mathrm{L}^{-1}$ ) y la maceración se hizo por espacio $5 \mathrm{~d}$. Luego se filtró en papel Whatman ${ }^{\circledR} \mathrm{N}^{\circ}$ ).

Para D. magna se utilizaron envases con 100 $\mathrm{mL}$ de la solución al $20 \%$ de concentración. Se utilizaron individuos-neonatos de hasta $24 \mathrm{~h}$ de edad (Iannacone et al., 2014). En cada envase se pusieron cinco individuos más un control sólo con agua potable reposada al menos una semana, con cuatro repeticiones. La toxicidad de los tratamientos se evaluó luego de $48 \mathrm{~h}$.

Para S. zeamais se pusieron $10 \mathrm{~g}$ de maíz remojados previamente 5 seg para cada repetición, sobre papel tissue ${ }^{\circledR}$ para el secado a temperatura ambiente. Luego se agregaron 10 individuos de cada especie en cada envase plástico con cuatro repeticiones más el control sólo con agua destilada. Las evaluaciones de mortalidad se hicieron cada $24 \mathrm{~h}$ durante $5 \mathrm{~d}(=120 \mathrm{~h})$, para determinar el efecto de los extractos acuoso.

Para C. koehleri se utilizaron larvas momificadas de $25 \mathrm{~d}$ desde la parasitación, y adultos de $\leq 24$ $\mathrm{h}$ de edad. Se realizaran dos pruebas de toxicidad, por contacto residual y por inmersión.

Toxicidad por contacto residual para adultos. Los adultos de la microavispa se utilizaron para un bioensayo con frascos de vidrio de $2,2 \mathrm{~cm}$ de radio y $4 \mathrm{~cm}$ de altura, de $12,5 \mu \mathrm{L}$, a los que se agregaron las concentraciones con una pipeta con puntas descartables, y luego se esparcieron homogéneamente con hisopos separados sobre la superficie interna. Los viales se secaron a temperatura ambiente por $2 \mathrm{~h}$ o alternativamente en estufa a $35^{\circ} \mathrm{C}$ durante 1 $\mathrm{h}$ con sus respetivos tapones de algodón. Los experimentos con C. koehleri se hicieron con adultos $<24 \mathrm{~h}$ de edad. Las microavispas se alimentaron hasta 2-3 h antes del tratamiento con solución de sacarosa 1:1. Se utilizaron machos y hembras indistintamente desde los frascos de emergencia de adultos. Estos se consideraron muertos cuando no se posaron sobre el vial y se encontraron en el fondo con las patas hacia arriba, durante $10 \mathrm{~s}$ de observación bajo aumento. Tanto el control como los tratamientos utilizaron agua reposada, con cuatro frascos (uno por repetición). Se usaron 20 adultos por cada sustancia evaluada y cinco adultos por repetición más el control. Para los ensayos de toxicidad aguda, los frascos se mantuvieron en condiciones de cría y oscuridad señaladas por Iannacone et al. (2007) y se observó la mortalidad acumulada a varias h desde la exposición. Las lecturas se hicieron siempre cuando la mortalidad en el control fue $<10 \%$. 
Toxicidad por inmersión. Las aplicaciones se hicieron sobre larvas momificadas de $P$. operculella parasitadas por $C$. koehleri por inmersión de $5 \mathrm{~s}$ en los extractos botánicos en placas Petri de plástico. Luego de la inmersión las larvas parasitadas se pusieron 10 min en papel tissue ${ }^{\circledR}$ para absorber el exceso de solución y se dejaron secar $1 \mathrm{~h}$ a temperatura ambiente. Se trataron 10 larvas parasitadas, que se pusieron en frascos de $1 \mathrm{~cm}$ de radio y $5 \mathrm{~cm}$ de altura (Iannacone et al., 2007). Las lecturas se hicieron hasta la emergencia cercana al $100 \%$ de los adultos de C. koehleri de las larvas momificadas de $P$. operculella en el control. El porcentaje de emergencia de C. koehleri se calculó contando el número de microavispas emergidas de una larva momificada de $P$. operculella, dividiéndolo por el número total de cámaras parasitadas (además, se contó el número de adultos formados no emergidos y el número de larvas y pupas de C. koehleri) (Iannacone et al., 2007).

Cocción e infusión acuosa. Se prepararon extractos acuosos por cocción de $20 \mathrm{~g}$ de muestra por $100 \mathrm{~mL}$ de agua destilada, durante $10 \mathrm{~min}$. Las infusiones de los extractos acuosos se prepararon con $20 \mathrm{~g}$ de muestra por $100 \mathrm{~mL}$ de agua destilada, hirviendo agua reposada a $100^{\circ} \mathrm{C}$ y una vez a esta temperatura se retiró el calor y se agregó inmediatamente los extractos hasta que enfriaron. El procedimiento de bioensayo fue el mismo que con la maceración.

Maceración con alcohol etílico. La extracción se hizo con alcohol etílico absoluto, desde una solución madre al $20 \%$, con maceración por $5 \mathrm{~d}$. El procedimiento de bioensayo fue el mismo que para la maceración, más un control extra sólo con alcohol etílico al $20 \%$ y otro con agua.

Repelencia. Los efectos de repelencia de los bioensayos contra $S$. zeamais se evaluaron usando el método del área preferente (McDonald et al., 1970), con papel filtro Whatman № 1 de 6,2 $\mathrm{cm}$ de radio cortado por la mitad $\left(120,76 \mathrm{~cm}^{2}\right)$. Se prepararon diluciones acuosas al 20\% de cada tratamiento por maceración, cocción e infusión, y por maceración en etanol. Cada solución se aplicó uniformemente a la mitad del papel filtro a la dosis mayor, y la otra mitad del papel para el control se trató con agua destilada. Los papeles filtros se airearon $10 \mathrm{~min}$ para evaporar el solvente, y se pegaron con cinta adhesiva en placas Petri. Luego se pusieron 20 gorgojos adultos no sexados en el centro de la placa, que se mantuvo en condiciones de cría y oscuridad señaladas por Iannacone et al. (2007), con cuatro repeticiones. Los gorgojos presentes, en el área control $(\mathrm{Nc})$ y en el área tratada $(\mathrm{Nt})$ se contaron a las $2 \mathrm{~h}$. El porcentaje de repelencia (PR) se determinó según la fórmula $\mathrm{PR}=[(\mathrm{Nc}-\mathrm{Nt}) /(\mathrm{Nc}$ $+\mathrm{Nt})]^{*} 100$. El porcentaje de repelencia de cada tratamiento se asignó de 0 a $\mathrm{V}$ clases de repelencia (Juliana y Su, 1983): clase $0(\mathrm{PR}<0,1 \%)$, clase I $(\mathrm{PR}<0,1-20 \%)$, clase II (PR $<20,1-40 \%)$, clase III (PR $<40,1-60 \%)$, clase IV (PR $<60,1-80 \%$ ) y clase $\mathrm{V}(\mathrm{PR}<80,1-100 \%)$. También se utilizó el Índice de Repelencia (IR), que clasifica al tratamiento como neutro si el índice es $=$ a 1 , atrayente si es >a 1 y repelente si es $<1$, según la fórmula IR $=2 \mathrm{G} / \mathrm{G}+\mathrm{P}$, donde $\mathrm{G}$ : porcentaje de insectos en el tratamiento, y P: porcentaje de insectos en el testigo. Se consideraron promisorios los tratamientos que presentaron $40 \%$ de mortalidad (Lagunes, 1994).

\section{Diseño experimental y tratamiento estadísti-} co. Para D. magna, las pruebas de toxicidad se evaluaron en cinco concentraciones $(1,25 ; 2,5 ; 5$; 10 y $20 \%$ de extractos de hojas, corteza y raíces en cuatro tratamientos: maceración, cocción e infusión en agua, y maceración en alcohol etílico, más el control, con cuatro repeticiones, en un diseño de 6 tratamiento en 4 bloques completamente aleatorizados. Las concentraciones letales medias $\left(\mathrm{CL}_{50} \mathrm{~s}\right)$ se calcularon con el software Probit versión 1,5 (USEPA, 1993).

Para S. zeamais y C. koehleri, las pruebas de toxicidad se hicieron a una sola concentración del $20 \%$ de extractos de hoja, corteza y raíces y cuatro tratamientos: maceración, cocción e infusión en agua, y maceración en alcohol etílico, más el control, con cuatro repeticiones. En el bioensayo con C. koehleri se evaluaron 13 tratamientos x 4 repeticiones en un diseño de bloques completamente aleatorizados. Para S. zeamais solamente se incluyó un tratamiento con polvos secos de hojas, corteza y raíces, con 16 tratamientos x 4 repeticiones. Para el ensayo por inmersión de adultos emergidos, no emergidos y formas larvarias y pupas no emergidas de $C$. koehleri se utilizó un ANDEVA completamente aleatorizado de 13 tratamientos $x 10$ repeticiones. Para estabilizar el error de la varianza, la eficacia de los tratamientos se evaluó a través de un modelo aditivo lineal, previa transformación por $\log (x+1)$ de los resultados de mortalidad de adultos (\%) de S. zeamais, de granos dañados (\%) y pérdida de peso (\%) del grano de maíz por $S$. zeamais, de la mortalidad de adultos (\%) de C. koehleri, de adultos emergidos, no emergidos y de larvas y pupas de C. koehleri). Los promedios de los tratamientos se separaron con la prueba de Tukey. Los análisis se hicieron con programa estadístico SPSS 21.0 para Windows XP (IBM, 2011). 


\section{RESULTADOS}

Daphnia magna. A $48 \mathrm{~h}$ de la exposición los $\mathrm{CL}_{50}$ para D. magna en $\mathrm{g} \mathrm{L}^{-1}$ indican que los cuatro tratamientos con mayor toxicidad fueron la infusión en agua de hojas, la cocción en agua de raíces, la cocción en agua de hojas y la maceración en agua de hojas (Tabla 1).
Sitophilus zeamais. No se encontraron diferencias significativas en la mortalidad de $S$. zeamais entre los 15 tratamientos en base a $S$. peruviana y el control a las 24 y 48 h desde la exposición (Tabla 2). A las $72 \mathrm{~h}$ de la exposición solo se encontraron diferencias en la mortalidad de S. zeamais entre la cocción de la corteza y el control. A las 96 y $120 \mathrm{~h}$ de la exposición, solo el polvo de

Tabla 1. Valores de $\mathrm{CL}_{50}$ de los extractos de hoja, corteza y raíz de Sambucus peruviana sobre la pulga de agua Daphnia magna a 48 h de exposición.

Table 1. $\mathrm{LC}_{50}$ values of leaf, bark and root extracts of Sambucus peruviana on the water flea Daphnia magna at $48 \mathrm{~h}$ of exposure.

\begin{tabular}{lr}
\hline Tratamientos & CL $_{50}$ \\
\hline Hojas maceradas en agua & g-L ${ }^{-1}$ \\
Hojas cocidas & 6,00 \\
Hojas en infusión & 5,15 \\
Hojas maceradas en alcohol & 0,44 \\
Corteza macerada en agua & 11,00 \\
Corteza cocida & 13,40 \\
Corteza en infusión & 22,80 \\
Corteza macerada en alcohol & 11,00 \\
Raíz macerada en agua & 8,00 \\
Raíz cocida & 12,11 \\
Raíz en infusión & 3,00 \\
Raíz macerada en alcohol & 8,94 \\
\hline
\end{tabular}

Tabla 2. Efecto tóxico de diferentes tratamientos de hoja, corteza y raíz de Sambucus peruviana sobre la mortalidad del gorgojo del maíz (Sitophilus zeamais) hasta 120 h de exposición.

Table 2. Toxic effect of different treatments of leaf, bark and root of Sambucus peruviana on the mortality of maize weevil (Sitophilus zeamais) up to $120 \mathrm{~h}$ of exposure.

\begin{tabular}{lccccc}
\hline Tratamientos & \multicolumn{5}{c}{ Mortalidad (\%) } \\
\cline { 2 - 6 } & $\mathbf{2 4} \mathbf{h}$ & $\mathbf{4 8} \mathbf{h}$ & $\mathbf{7 2} \mathbf{h}$ & $\mathbf{9 6} \mathbf{h}$ & $\mathbf{1 2 0} \mathbf{h}$ \\
\hline Blanco con agua & $0,00 \pm 0,00 \mathrm{a}$ & $0,00 \pm 0,00 \mathrm{a}$ & $0,00 \pm 0,00 \mathrm{a}$ & $0,00 \pm 0,00 \mathrm{a}$ & $0,00 \pm 0,00 \mathrm{a}$ \\
Hojas secas en polvo & $0,00 \pm 0,00 \mathrm{a}$ & $0,00 \pm 0,00 \mathrm{a}$ & $0,00 \pm 0,00 \mathrm{a}$ & $0,00 \pm 0,00 \mathrm{a}$ & $0,00 \pm 0,00 \mathrm{a}$ \\
Hojas maceradas en agua & $0,00 \pm 0,00 \mathrm{a}$ & $0,00 \pm 0,00 \mathrm{a}$ & $0,00 \pm 0,00 \mathrm{a}$ & $0,00 \pm 0,00 \mathrm{a}$ & $5,00 \pm 0,50 \mathrm{a}$ \\
Hojas cocidas & $0,00 \pm 0,00 \mathrm{a}$ & $2,50 \pm 0,25 \mathrm{a}$ & $5,00 \pm 0,28 \mathrm{ab}$ & $5,00 \pm 0,28 \mathrm{a}$ & $17,5 \pm 0,47 \mathrm{a}$ \\
Hojas en infusión & $0,00 \pm 0,00 \mathrm{a}$ & $0,00 \pm 0,00 \mathrm{a}$ & $2,50 \pm 0,25 \mathrm{ab}$ & $2,50 \pm 0,25 \mathrm{a}$ & $2,50 \pm 0,25 \mathrm{a}$ \\
Hojas maceradas en alcohol & $0,00 \pm 0,00 \mathrm{a}$ & $5,00 \pm 0,50 \mathrm{a}$ & $5,00 \pm 0,50 \mathrm{ab}$ & $7,50 \pm 0,75 \mathrm{a}$ & $7,50 \pm 0,75 \mathrm{a}$ \\
Corteza seca en polvo & $0,00 \pm 0,00 \mathrm{a}$ & $0,00 \pm 0,00 \mathrm{a}$ & $5,00 \pm 0,28 \mathrm{ab}$ & $27,50 \pm 0,63 \mathrm{~b}$ & $27,50 \pm 0,63 \mathrm{~b}$ \\
Corteza macerada en agua & $0,00 \pm 0,00 \mathrm{a}$ & $5,00 \pm 0,28 \mathrm{a}$ & $7,50 \pm 0,48 \mathrm{ab}$ & $10,00 \pm 0,70 \mathrm{a}$ & $10,00 \pm 0,70 \mathrm{a}$ \\
Corteza cocidas & $0,00 \pm 0,00 \mathrm{a}$ & $0,00 \pm 0,00 \mathrm{a}$ & $12,50 \pm 0,25 \mathrm{~b}$ & $15,00 \pm 0,28 \mathrm{ab}$ & $15,00 \pm 0,28 \mathrm{ab}$ \\
Corteza en infusión & $0,00 \pm 0,00 \mathrm{a}$ & $0,00 \pm 0,00 \mathrm{a}$ & $0,00 \pm 0,00 \mathrm{a}$ & $0,00 \pm 0,00 \mathrm{a}$ & $0,00 \pm 0,00 \mathrm{a}$ \\
Corteza macerada en alcohol & $0,00 \pm 0,00 \mathrm{a}$ & $0,00 \pm 0,00 \mathrm{a}$ & $0,00 \pm 0,00 \mathrm{a}$ & $0,00 \pm 0,00 \mathrm{a}$ & $5,00 \pm 0,50 \mathrm{a}$ \\
Raíz seca en polvo & $0,00 \pm 0,00 \mathrm{a}$ & $5,00 \pm 0,50 \mathrm{a}$ & $7,50 \pm 0,48 \mathrm{ab}$ & $7,50 \pm 0,48 \mathrm{a}$ & $12,50 \pm 0,95 \mathrm{a}$ \\
Raíz maceradas en agua & $0,00 \pm 0,00 \mathrm{a}$ & $0,00 \pm 0,00 \mathrm{a}$ & $0,00 \pm 0,00 \mathrm{a}$ & $0,00 \pm 0,00 \mathrm{a}$ & $0,00 \pm 0,00 \mathrm{a}$ \\
Raíz cocidas & $0,00 \pm 0,00 \mathrm{a}$ & $0,00 \pm 0,00 \mathrm{a}$ & $0,00 \pm 0,00 \mathrm{a}$ & $0,00 \pm 0,00 \mathrm{a}$ & $0,00 \pm 0,00 \mathrm{a}$ \\
Raíz en infusión & $0,00 \pm 0,00 \mathrm{a}$ & $0,00 \pm 0,00 \mathrm{a}$ & $0,00 \pm 0,00 \mathrm{a}$ & $0,00 \pm 0,00 \mathrm{a}$ & $0,00 \pm 0,00 \mathrm{a}$ \\
Raíz maceradas en alcohol & $0,00 \pm 0,00 \mathrm{a}$ & $0,00 \pm 0,00 \mathrm{a}$ & $0,00 \pm 0,00 \mathrm{a}$ & $0,00 \pm 0,00 \mathrm{a}$ & $0,00 \pm 0,00 \mathrm{a}$ \\
\hline
\end{tabular}

Promedio \pm error estándar.

Promedios en la misma fila con la misma letra no difieren significativamente $(P \leq 0,05)$, según test de Tukey. 
corteza presentó diferencias significativas con el control. Todos los tratamientos con raíces y hojas no tuvieron efecto en la mortalidad de S. zeamais. Los porcentajes de granos dañados de los tratamientos por maceración, cocción e infusión en agua de la corteza, presentaron los mayores porcentajes de granos dañados, con diferencias significativas con el control (Tabla 3). No se encontraron pérdidas significativas de peso del grano por S. zeamais entre todos los tratamientos en comparación con el control. Las pérdidas de grano de maíz fueron $<$ al 3\% de peso. Ninguno de los tratamientos tuvo una repelencia hacia $S$. zeamais mayor al nivel II (PR $<20,1-40 \%$ ), clase que sólo se observó en la maceración en etanol de hojas y raíces (Tabla 4). Tres tratamientos fueron atrayentes $(25 \%)$ y nueve repelentes $(75 \%)$. El tratamiento más repelente fue el de maceración en etanol de raíces.

Copidosoma koehleri. El ensayo por contacto residual en adultos de $C$. koehleri presentó diferencias entre la infusión de raíces y el control entre 3 y $48 \mathrm{~h}$ desde la exposición (Tabla 5). También a las $48 \mathrm{~h}$ de exposición, los extractos de maceración en etanol de la corteza y en agua de raíces causaron mortalidades de C. koehleri mayores al control.
También se encontraron diferencias entre los tratamientos de los adultos emergidos con maceración en agua de la corteza, infusión en agua de raíces, maceración en agua de raíces y maceración en agua de hojas y el control (Tabla 6).

\section{DISCUSIÓN}

Daphnia magna. El rango de $\mathrm{CL}_{50}$ observado para D. magna con los extractos de S. peruviana en el presente trabajo fue amplio $(0,44-22,8 \mathrm{~g}$ $\left.\mathrm{L}^{-1}\right)$. El $\mathrm{CL}_{50}$ menor a las $48 \mathrm{~h}$ se obtuvo con la infusión de hojas. Este crustáceo se ha utilizado para evaluar muchas sustancias en ambientes acuáticos en Perú (Núñez y Hurtado, 2005; Iannacone et al., 2008). La actividad en estos ambientes de los extractos vegetales se relaciona con algunos factores intrínsecos y extrínsecos, tales como la especie y variedad de la planta, período de recolección, parte cosechada y forma de preparación, extracción y uso en el campo y calidad físico-química del recurso acuático (Iannacone et al., 2007; 2008).

Sitophilus zeamais. A 96 y 120 h desde la exposición, sólo el polvo de corteza presentó diferencias significativas en la mortalidad de $S$. zeamais

Tabla 3. Granos dañados y pérdida de peso del grano de maíz por gorgojo del maíz (Sitophilus zeamais) con tratamientos de hoja, corteza y raíz de Sambucus peruviana hasta $120 \mathrm{~h}$ de exposición.

Table 3. Damaged grains and weight loss of corn grain by maize weevil (Sitophilus zeamais) under different treatments of leaf, bark and root of Sambucus peruviana up to $120 \mathrm{~h}$ of exposure.

\begin{tabular}{lcc}
\hline Tratamientos & Granos dañados & Pérdida de peso del grano \\
\hline & & $\%$ \\
Blanco con agua & $0,78 \pm 0,07 \mathrm{a}$ & $1,56 \pm 0,17 \mathrm{a}$ \\
Hojas secas en polvo & $0,42 \pm 0,08 \mathrm{a}$ & $0,95 \pm 0,66 \mathrm{a}$ \\
Hojas maceradas en agua & $0,49 \pm 0,15 \mathrm{a}$ & $0,95 \pm 0,02 \mathrm{a}$ \\
Hojas cocidas & $0,42 \pm 0,10 \mathrm{a}$ & $2,32 \pm 1,04 \mathrm{a}$ \\
Hojas en infusión & $0,38 \pm 0,06 \mathrm{a}$ & $0,85 \pm 0,58 \mathrm{a}$ \\
Hojas maceradas en alcohol & $0,41 \pm 0,06 \mathrm{a}$ & $0,75 \pm 0,25 \mathrm{a}$ \\
Corteza seca en polvo & $0,74 \pm 0,21 \mathrm{a}$ & $0,50 \pm 0,28 \mathrm{a}$ \\
Corteza macerada en agua & $2,32 \pm 0,34 \mathrm{bc}$ & $2,43 \pm 0,87 \mathrm{a}$ \\
Corteza cocida & $2,51 \pm 0,40 \mathrm{c}$ & $2,75 \pm 0,27 \mathrm{a}$ \\
Corteza en infusión & $2,61 \pm 0,23 \mathrm{c}$ & $1,25 \pm 0,24 \mathrm{a}$ \\
Corteza macerada en alcohol & $0,49 \pm 0,15 \mathrm{a}$ & $0,98 \pm 0,01 \mathrm{a}$ \\
Raíz seca en polvo & $0,81 \pm 0,15 \mathrm{a}$ & $1,17 \pm 0,53 \mathrm{a}$ \\
Raíz macerada en agua & $1,32 \pm 0,21 \mathrm{abc}$ & $1,39 \pm 0,53 \mathrm{a}$ \\
Raíz cocida & $0,58 \pm 0,09 \mathrm{a}$ & $0,88 \pm 0,03 \mathrm{a}$ \\
Raíz en infusión & $0,57 \pm 0,09 \mathrm{a}$ & $0,88 \pm 0,03 \mathrm{a}$ \\
Raíz macerada en alcohol & $1,13 \pm 0,49 \mathrm{ab}$ & $2,13 \pm 2,13 \mathrm{a}$ \\
\hline
\end{tabular}

Promedio \pm error estándar.

Promedios en la misma fila con la misma letra no difieren significativamente $(\mathrm{P} \leq 0,05)$, según test de Tukey. 
Tabla 4. Índice, porcentaje y clase de repelencia de los tratamientos en base a Sambucus peruviana sobre el gorgojo del maíz.

Table 4. Index, percentage, and repellence class of the treatments with Sambucus peruviana on the maize weevil.

\begin{tabular}{lccc}
\hline Tratamientos & Índice de repelencia & Repelencia (\%) & Clase de repelencia \\
\hline Hojas maceradas en agua & $0,92 \mathrm{R}$ & 7,73 & $\mathrm{I}$ \\
Hojas cocidas & $1,18 \mathrm{~A}$ & $-17,70$ & 0 \\
Hojas en infusión & $1,10 \mathrm{~A}$ & $-10,00$ & 0 \\
Hojas maceradas en alcohol & $0,80 \mathrm{R}$ & 30,00 & $\mathrm{II}$ \\
Corteza maceradas en agua & $0,80 \mathrm{R}$ & 19,20 & $\mathrm{I}$ \\
Corteza cocida & $0,95 \mathrm{R}$ & 5,13 & 0 \\
Corteza en infusión & $0,92 \mathrm{R}$ & 2,74 & 0 \\
Corteza macerada en alcohol & $0,92 \mathrm{R}$ & 7,73 & $\mathrm{I}$ \\
Raíz macerada en agua & $1,15 \mathrm{~A}$ & $-15,00$ & 0 \\
Raíz cocida & $0,95 \mathrm{R}$ & 5,00 & 0 \\
Raíz en infusión & $0,95 \mathrm{R}$ & 4,10 & 0 \\
Raíz macerada en alcohol & $0,65 \mathrm{R}$ & 35,00 & $\mathrm{II}$ \\
\hline
\end{tabular}

IR = Índice de Repelencia: 1 = neutro; IR $>1$ atrayente; IR $<1$ repelente.

Tabla 5. Efectos tóxicos por contacto residual de los extractos de hoja, corteza y raíz de Sambucus peruviana sobre adultos de Copidosoma koehleri de $3 \mathrm{~h}$ a $48 \mathrm{~h}$ de exposición.

Table 5. Residual toxic effects by contact of leaf, bark and root extracts of Sambucus peruviana on adults of Copidosoma koehleri from $3 \mathrm{~h}$ to $48 \mathrm{~h}$ exposure.

\begin{tabular}{lcccc}
\hline Tratamientos & \multicolumn{4}{c}{ Mortalidad } \\
\cline { 2 - 5 } & $\mathbf{3 ~ h}$ & $\mathbf{1 2} \mathbf{~ h}$ & $\mathbf{2 4} \mathbf{~ h}$ & $\mathbf{4 8 ~ h}$ \\
\hline Blanco con agua & $---00 \mathrm{a}$ & $0,00 \mathrm{a}$ & $0,00 \mathrm{a}$ & $0,00 \mathrm{a}$ \\
Hojas maceradas en agua & $0,00 \mathrm{a}$ & $5,00 \mathrm{a}$ & $7,50 \mathrm{ab}$ & $7,50 \mathrm{ab}$ \\
Hojas cocidas & $0,00 \mathrm{a}$ & $0,00 \mathrm{a}$ & $2,50 \mathrm{ab}$ & $2,50 \mathrm{ab}$ \\
Hojas en infusión & $0,00 \mathrm{a}$ & $0,00 \mathrm{a}$ & $0,00 \mathrm{a}$ & $2,50 \mathrm{ab}$ \\
Hojas maceradas en alcohol & $0,00 \mathrm{a}$ & $0,00 \mathrm{a}$ & $2,50 \mathrm{ab}$ & $2,50 \mathrm{ab}$ \\
Corteza macerada en agua & $0,00 \mathrm{a}$ & $2,50 \mathrm{a}$ & $10,00 \mathrm{abc}$ & $10,00 \mathrm{abc}$ \\
Corteza cocida & $0,00 \mathrm{a}$ & $15,00 \mathrm{ab}$ & $15,00 \mathrm{abc}$ & $15,00 \mathrm{abc}$ \\
Corteza en infusión & $2,50 \mathrm{a}$ & $15,00 \mathrm{ab}$ & $15,00 \mathrm{abc}$ & $15,00 \mathrm{abc}$ \\
Corteza macerada en alcohol & $0,00 \mathrm{a}$ & $0,00 \mathrm{a}$ & $2,50 \mathrm{ab}$ & $2,50 \mathrm{ab}$ \\
Raíz macerada en agua & $0,00 \mathrm{a}$ & $12,50 \mathrm{ab}$ & $17,50 \mathrm{bc}$ & $17,50 \mathrm{bc}$ \\
Raíz cocida & $0,00 \mathrm{a}$ & $0,00 \mathrm{a}$ & $0,00 \mathrm{a}$ & $0,00 \mathrm{a}$ \\
Raíz en infusión & $20,00 \mathrm{~b}$ & $25,00 \mathrm{~b}$ & $25,00 \mathrm{c}$ & $25,00 \mathrm{c}$ \\
Raíz macerada en alcohol & $0,00 \mathrm{a}$ & $0,00 \mathrm{a}$ & $0,00 \mathrm{a}$ & $0,00 \mathrm{a}$ \\
\hline
\end{tabular}

Promedios en la misma fila con la misma letra no difieren significativamente $(\mathrm{P} \leq 0,05)$, según test de Tukey.

en comparación con el control, aunque no sobrepasó el $30 \%$. Para que una planta con propiedades insecticidas sea promisoria para el control de plagas de granos almacenados la mortalidad debe ser $>$ al 40\% (Lagunes, 1994; Akob y Ewete, 2007). Aunque en todos los tratamientos, la mortalidad de $S$. zeamais aumentó con el tiempo de exposición, al igual que en Silva et al. (2003) y Tapondjou et al. (2005), los resultados para el control de plagas no se consideran auspiciosos para el uso de esta planta como un insecticida botánico contra plagas de granos almacenados.

Aunque la mayoría de los bioensayos con $S$. zeamais se hacen con hojas pues éstas concentran los compuestos activos (Torres et al., 2015), muchos insecticidas de origen vegetal se obtie- 
Tabla 6. Efecto tóxico de tratamientos en base a Sambucus peruviana por inmersión de estados de desarrollo de Copidosoma koehleri.

Table 6. Toxic effect of different treatments with Sambucus peruviana by immersion of Phthorimaea operculella mummified larvae on Copidosoma koehleri.

\begin{tabular}{lccc}
\hline Tratamientos & $\begin{array}{c}\text { Adultos } \\
\text { emergidos }\end{array}$ & $\begin{array}{c}\text { Adultos } \\
\text { no emergidos }\end{array}$ & $\begin{array}{c}\text { Larvas y pupas } \\
\text { no emergidas }\end{array}$ \\
\hline Blanco con agua & ----------- \\
Hoja maceradas en agua & $100,00 \pm 0,00 \mathrm{~d}$ & $0,00 \pm 0,00 \mathrm{a}$ & $0,00 \pm 0,00 \mathrm{a}$ \\
Hojas cocidas & $30,39 \pm 5,84 \mathrm{a}$ & $2,89 \pm 1,61 \mathrm{a}$ & $66,76 \pm 6,69 \mathrm{~d}$ \\
Hoja en infusión & $15,74 \pm 10,63 \mathrm{a}$ & $31,77 \pm 12,72 \mathrm{~b}$ & $52,47 \pm 13,78 \mathrm{bcd}$ \\
Hoja maceradas en alcohol & $44,43 \pm 13,78 \mathrm{abc}$ & $25,27 \pm 10,38 \mathrm{ab}$ & $30,28 \pm 13,45 \mathrm{abcd}$ \\
Corteza macerada en agua & $89,01 \pm 4,83 \mathrm{~cd}$ & $5,74 \pm 2,52 \mathrm{ab}$ & $5,24 \pm 3,29 \mathrm{ab}$ \\
Corteza cocida & $37,11 \pm 8,42 \mathrm{ab}$ & $0,72 \pm 0,37 \mathrm{a}$ & $62,16 \pm 8,61 \mathrm{~d}$ \\
Corteza en infusión & $32,06 \pm 13,71 \mathrm{a}$ & $5,20 \pm 2,49 \mathrm{a}$ & $62,72 \pm 14,85 \mathrm{~d}$ \\
Corteza maceradas en alcohol & $54,81 \pm 12,67 \mathrm{abcd}$ & $14,18 \pm 6,57 \mathrm{ab}$ & $30,99 \pm 14,32 \mathrm{abcd}$ \\
Raíz macerada en agua & $91,33 \pm 3,75 \mathrm{~d}$ & $4,72 \pm 1,96 \mathrm{a}$ & $3,94 \pm 2,31 \mathrm{ab}$ \\
Raíz cocida & $32,07 \pm 4,26 \mathrm{a}$ & $2,50 \pm 2,50 \mathrm{a}$ & $65,41 \pm 2,56 \mathrm{~d}$ \\
Raíz en infusión & $33,58 \pm 13,69 \mathrm{a}$ & $7,88 \pm 3,54 \mathrm{ab}$ & $58,53 \pm 15,77 \mathrm{~cd}$ \\
Raíz maceradas en alcohol & $29,90 \pm 12,60 \mathrm{a}$ & $9,96 \pm 4,91 \mathrm{ab}$ & $60,12 \pm 14,58 \mathrm{~d}$ \\
\hline
\end{tabular}

Promedio \pm error estándar.

Promedios en la misma fila con la misma letra no difieren significativamente $(P \leq 0,05)$, según test de Tukey.

nen también de tallos, raíces, flores y semillas (Issakul et al., 2011; Fedaku et al., 2012). En el presente estudio el tratamiento de corteza en polvo causó la mayor mortalidad de S. zeamais, incluso después del tiempo establecido para los bioensayos (55\% a las $196 \mathrm{~h}$ ), resultado que indica que recién después de ese período desde la exposición se obtendrían resultados que armonizarían con la propuesta de Lagunes (1994) de mortalidad > de $40 \%$ para ser considerado un tratamiento botánico promisorio. Se ha observado mortalidad significativa de $S$. zeamais por acción de polvos secos de diversas plantas a bajas concentraciones (Silva et al., 2003; 2005; Salvadores et al., 2007). El polvo de la corteza del saúco pudiera estar actuando sobre $S$. zeamais como veneno estomacal y barrera física (Fedaku et al., 2012). Así, según los criterios de Nuñez et al. (2010), para proteger $100 \mathrm{~kg}$ de semillas de cereal se requerirían $16 \mathrm{~kg}$ de polvo y cerca de 20 $\mathrm{kg}$ de corteza de S. peruviana. Esta concentración parecería impráctica, si no se mezcla con otros compuestos químicos en polvo como el carbonato de calcio (Nuñez et al., 2010).

En otro trabajo con polvo de hojas de S. peruviana, Iannacone et al. (2005) no obtuvieron mortalidad de $S$. zeamais hasta con una concentración máxima de $20 \%$. Luego, Iannacone et al. (2006) hicieron ensayos con extractos etanólicos de frutos maduro y verdes de S. peruviana, y tampoco obtuvieron mortalidad de $S$. zeamais, aunque las hojas y los frutos de saúcos evaluados se obtuvieron en el distrito de Pueblo Libre, Lima, Perú (entre 85 y $90 \mathrm{msnm}$ ), mientras que S. peruviana es una especie de zonas altoandinas (entre 2000 y $4000 \mathrm{msnm}$ ), y el cambio de ambiente puede producir menos metabolitos que aquellas plantas que se encuentran en su hábitat natural. Nuñez et al. (2010) y Pinto et al. (2016) indican que la variabilidad natural de los principios activos o metabolitos secundarios principales con propiedades insecticidas de las plantas dependen de la zona geográfica, estado fenológico y época del año. Otras plantas evaluadas sí han resultado tóxicas o repelentes (Iannacone et al., 2015).

El porcentaje de granos dañados de los tratamientos de maceración y cocción en agua, e infusión en agua de la corteza fue mayor que en el testigo, resultados que indican una ligera atracción de $S$. zeamais al grano de maíz con estos tres extractos, los que así no serían utilizables como repelentes. Al evaluar los resultados obtenidos con papel filtro, los tratamientos que fueron atrayentes para $S$. zeamais fueron la cocción e infusión de hojas y la maceración de raíces de $S$. peruviana. Los resultados de repelencia de S. zeamais frente a los extractos fueron diferentes entre los granos almacenados de maíz y el papel filtro.

La pérdida de peso del grano por acción de S. zeamais fue muy escasa (3\%) y sin diferencias entre los tratamientos y el control. Los efectos tóxicos o repelentes de los fitoquímicos hacia $S$. 
zeamais dependen de numerosos factores, entre los que se encuentran la composición química y la susceptibilidad del insecto (Cruz et al., 2011). Según la escala de Juliana y Su (1983), el poder repelente del saúco es muy bajo, no mayor al nivel II (Tapondjou et al., 2005). En general, en nuestro estudio los extractos no tuvieron efectos repelentes significativos contra el gorgojo del maíz, a pesar que el $75 \%$ de los tratamientos en Pinto et al. (2016) presentó un índice de repelencia $<1$.

Silva et al. (2003) recomiendan voltear una vez al mes los envases de almacenamiento para homogeneizar el polvo entre los granos de maíz y favorecer el control del gorgojo del maíz. El uso de extractos y polvos del saúco, una planta endémica de la zona andina, sería una alternativa de prevención cara y poco sostenible como insecticida y repelente para el Perú, debido a que las cantidades necesarias para su uso masivo podrían afectar a esta especie en su ambiente natural.

Copidosoma koehleri. La infusión y maceración en agua de raíces de $S$. peruviana produjeron efectos residuales por contacto en adultos de $C$. koehleri a 48 h de la exposición. Según Iannacone et al. (2015), los resultados obtenidos en bioensayos ecotoxicológicos con plaguicidas en laboratorio sobre enemigos naturales que no son el objetivo del control químico sirven como referencia para orientar la selección de ellos en programas de manejo integrado de plagas. Aunque los tratamientos no superaron el $25 \%$ de mortalidad de la microavispa C. koehleri, el uso en control de plagas de los extractos en infusión y maceración en agua de raíces no sería muy compatible con el control biológico en caso de ser éste utilizado en MIP, a pesar que los componentes fitoquímicos del saúco no dañarían muy pronunciadamente a los adultos de este parasitoide. En comparación, extractos hexánicos de Schinus molle L. y Lantana camara L. han causado una mortalidad de C. koehleri $>50 \%$ a las $48 \mathrm{~h}$ de la exposición (Iannacone y Lamas, 2003).

Sin embargo, al evaluar los efectos subletales como la emergencia de adultos y en las larvas y pupas no emergidas de C. koehleri con hoja, los tratamientos en base a extractos de saúco podrían causar un efecto dañino. Aunque las larvas y pupas del parasitoide se encuentran protegidas por el cuerpo momificado de la larva hospedera, algunos principios activos pueden penetrar en su interior y causar la muerte del parasitoide en sus fases larvarias y de pupa de desarrollo, que tienen una cutícula relativamente aún no endurecida (Longley, 1999).

Los resultados con 12 preparados en base al saúco sobre $D$. magna y el parasitoide $C$. koehleri pueden ser ventajosos para las evaluaciones regulatorias en un posible registro de S. peruviana como plaguicida botánico en el Perú y para la selección de formulaciones con efectos plaguicidas para su integración holística en el MIP (Iannacone et al., 2006). Sin embargo, se requiere más investigación para evaluar los efectos secundarios contra las fases del ciclo biológico de ambos invertebrados, así como determinar el efecto de estos preparados de S. peruviana en la germinación de granos almacenados.

\section{CONCLUSIONES}

El polvo de corteza y la cocción en agua de corteza de S. peruviana debido a su efecto insecticida y repelente fueron los tratamientos más efectivos contra S. zeamais, y los extractos de hojas y raíces presentaron los mayores efectos en el ámbito acuático representado por D. magna y en el control biológico por C. koehleri.

\section{LITERATURA CITADA}

Akob, C.A., and F.K. Ewete. 2007. The efficacy of ashes of four locally used plant materials against Sithopilus zeamais (Coleoptera: Curculionidae) in Cameroon. International Journal of Tropical Insect Science 27:21-26.

Alvarado-Chávez, B. 2007. Plantas medicinales de la Cordillera Negra. Revista de la Academia Peruana de Salud 14:53-63.

APHA. 2012. Standard methods for the examination of water and wastewater. $22^{\text {th }}$ ed. American Public Health Association (APHA), American Water Works Association (AWWA), and Water Environment Federation (WEF), Washington D.C., USA.

Cruz, A., C. Rodríguez, y C. Ortiz. 2011. Efecto insecticida in vitro del extracto etanólico de algunas plantas sobre la mosca adulta Haematobia irritans. Revista Cubana de Plantas Medicinales 16:216-226. (en línea)

Díaz, M.C., Y. Pica, y A. Ronco. 2004. Bioensayo de toxicidad aguda con Daphnia magna. En G. Castillo (ed.). p. 52. Ensayos toxicológicos y métodos de evaluación de calidad de aguas. Estandarización, intercalibración, resultados y aplicaciones. Centro Internacional de Investigaciones para el Desarrollo, Ottawa, Canadá.

Fekadu, G., S. Waktole, and D.R. Santiago. 2012. Evaluation of plant powders and cooking oils against maize weevil, Sitophilus zeamais M. (Coleoptera: Curculionidae) under laboratory conditions. Molecular Entomology 3:4-14 
Iannacone, J., y L. Alvariño. 2010. Toxicidad en Schinus molle L. (Anacardiaceae) a cuatro controladores biológicos de plagas agrícolas en el Perú. Acta Zoológica Mexicana 26:603-615.

Iannacone, J, L. Alvariño, H. Ayala, y N. Salazar. 2008. Toxicidad de nueve plantas y de la cipermetrina en gorgojos de productos almacenados y en la pulga del agua Daphnia magna en el Perú. Biotempo 8:26-34.

Iannacone, J., L. Alvariño, M.I. La Torre, A. Guabloche, K. Ventura, J. Chero, et al. 2015. Toxicidad aguda y crónica de Tagetes elliptica (Asteraceae) y dimetoato sobre depredadores y parasitoides de plagas de importancia agrícola en Perú. The Biologist (Lima) 13:329-347.

Iannacone, J., L. Alvariño, J.C. Soto, y C. Salcedo. 2007. Efecto toxicológico del "Sachayoco", Paullinia clavigera (Sapindaceae) sobre Daphnia magna y sobre dos controladores biológicos de plagas agrícolas. Journal of the Brazilian Society of Ecotoxicology 2:15-25.

Iannacone, J., H. Ayala, L. Alvariño, E.C. Paredes, W. Villegas, J. Alomia, et al. 2014. Riesgo ecotoxicológico acuático y terrestre del bioplaguicida catahua, Hura crepitans (Euphorbiaceae). Revista de Toxicología 30:50-62.

Iannacone, J.A., H. Ayala, A. Román, R. Carrillo, J.C. Soto, C. Salcedo, et al. 2006. Efecto insecticida de cinco plantas sobre el gorgojo del maíz Sitophilus zeamais Motschulsky, 1855. Scientia 8:197-206.

Iannacone, J., H. Ayala, J. Álvarez, O. Leyva, y E. Bajalque. 2004. Cuatro plantas biocidas sobre Sitophilus zeamais y Stegobium paniceum en el Perú. Wiñay Yachay (Perú) 8:16-27.

Iannacone, J., H. Ayala, y A. Román. 2005. Efectos toxicológicos de cuatro plantas sobre el gorgojo del maíz Sitophilus zeamais Motschulsky 1855 (Coleoptera: Curculionidae) y sobre el gorgojo de las galletas Stegobium paniceum (Linnaeus 1761) (Coleoptera: Anobiidae) en Perú. Gayana 69:234-240.

Iannacone, J., y G. Lamas. 2003. Efectos toxicológicos de extractos de molle (Schinus molle) y lantana (Lantana camara) sobre Chrysoperla externa (Neuroptera: Chrysopidae), Trichogramma pintoi (Hymenoptera: Trichogrammatidae) y Copidosoma koehleri (Hymenoptera: Encyrtidae) en el Perú. Agricultura Técnica (Chile) 63:347-360.

Iannacone, J., y C. Quispe. 2004. Efecto insecticida de dos extractos vegetales sobre el gorgojo del maíz Sitophilus zeamais Motschulsky, 1855 (Coleoptera: Curculionidae), en el Perú. Revista Peruana de Entomología 44:81-87.

IBM. 2011. Corp. Released IBM SPSS Statistics for Windows. Version 20.0. IBM Corp., Armonk, New York, USA.
Issakul, K., J. Araya, E. Pawelzik, and C. Jatisatienr. 2011. Potential of Mammea siamensis as a botanical insecticide: Its efficiency on diamondback moth and side effects on non-target organisms. Journal of Medicinal Plants Research 5:2149-2156.

Juárez-Flores, B.I., Y. Jasso-Pineda, J.R. AguirreRivera, y I. Jasso-Pineda. 2010. Efecto de polvos de asteráceas sobre el gorgojo del maíz (Sitophilus zeamais Motsch). Polibotánica 30:123-135.

Juliana, G., and H.C.F. Su. 1983. Laboratory studies on several plant materials as insects repelents for protection of cereal grains. Journal of Economic Entomology 76:154-157.

Keasar, T., and S. Steinberg. 2008. Evaluation of the parasitoid Copidosoma koehleri for biological control of the potato tuber moth, Phthorimaea operculella, in Israeli potato fields. Biocontrol Science and Technology 18:325-336.

Lagunes, T.A. 1994. Extractos, polvos vegetales y polvos minerales para el combate de plagas del maíz y del frijol en la agricultura de subsistencia. 35 p. Memorias del Colegio de Postgraduados USAID-CONACYT-BORUCONSA, Montecillo, Texcoco, México.

Longley, M. 1999. A review of pesticide effects upon immature parasitoids within mummified hosts. International Journal of Pest Management 45:139-145.

McDonald, L.L., R.H. Guy, and R.D. Speirs. 1970. Preliminary evaluation of new candidate materials as toxicants, repellents and attractants against stored products insects. Marketing Research Report № 882. Agricultural Research Service, United States Department of Agriculture (USDA-ARS), Washington DC, USA.

Natividad-Bardales, A.D., R.M. Rojas-Portal, y R.L. Huaman-Leandro. 2014. Extracción de antocianinas del sauco (Sambucus peruviana H.B. \& K.) cultivado en Huánuco para uso agroindustrial. Revista de Investigación Agroindustrial 1:37-53.

Nuñez, M., y J. Hurtado. 2005. Bioensayos de toxicidad aguda utilizando Daphnia magna Straus (Cladocera, Daphniidae) desarrollada en medio de cultivo modificado. Revista Peruana de Biología 12:165-170.

Nuñez, O.P., G. Silva, V.M. Tapia, R.G. Hepp, J.C. Rodríguez-Maciel, y A. Lagunes-Tejeda. 2010. Toxicidad de polvos de follaje de paico (Chenopodium ambrosioides L.) y boldo (Peumus boldus Mol.) solos y en mezcla con carbonato de calcio sobre gorgojo del maíz (Sitophilus zeamais Motschulsky). Agro-Ciencia 26:71-80. 
Pinto, J.J., G. Silva, I. Figueroa, M. Tapia, A. Urbina, J.C. Rodríguez, and A. Lagunes. 2016. Insecticidal activity of powder and essential oil of Cryptocarya alba (Molina) Looser against Sitophilus zeamais Motschulsky. Chilean Journal of Agricultural Research 76:48-54.

Rodríguez-Cobos, A.C., y J. Iannacone. 2012. Resistencia de granos de variedades de maíz amarillo duro a Sitophilus zeamais Mostchulsky, 1855 (Coleoptera: Curculionidae) en el Perú. Revista Peruana Entomología 47:1-6.

Ruiz, R.S.G., C.E. Venegas, R.D. Ruidías, A.L. Horna, y C.C.W. Wilfredo. 2013. Capacidad antioxidante in vitro de los flavonoides totales obtenidos de las hojas de Sambucus peruviana H.B.K. (saúco) proveniente de la ciudad de Huamachuco. Revista Pharmaciencia 1:57-64.

Salvadores, Y., G. Silva, M. Tapia, y R. Hepp. 2007. Polvos de especias aromáticas para el control del gorgojo del maíz, Sitophilus zeamais Motschulsky, en trigo almacenado. Agricultura Técnica (Chile) 67:147-154.

Segoli, M., A. Bouskila, A.R. Harari, and T. Keasar. 2009. Developmental patterns in the polyembryonic parasitoid wasp Copidosoma koehleri. Arthropod Structure \& Development 38:84-90.
Silva A., G.I., R. Kiger-Melivilu, R. Hepp-Gallo, y M. Tapia-Vargas. 2005. Control de Sitophilus zeamais con polvos vegetales de tres especies del género Chenopodium. Pesquisa Agropecuária Brasileira 40:953-960.

Silva, G., D. Pizarro, P. Casals, y M. Berti. 2003. Evaluación de plantas medicinales en polvo para el control de Sitophilus zeamais Motschulsky en maíz almacenado. Revista Brasileira de Agrociencia 9:383-388.

Tapondjou, A.L., C. Adler, D.A. Fontem, H. Bouda, and C. Reichmuth. 2005. Bioactivities of cymol and essential oils of Cupressus sempervivens and Eucaliptus saligna against Sitophilus zeamais Motschulsky and Tribolium confusum du Val. Journal of Stored Product Research 41:91-102.

Torres, C., G. Silva, M. Tapia, J.C. Rodríguez, A. Urbina, I. Figueroa, et al. 2015. Propiedades insecticidas del polvo de Laurelia sempervirens L. para el control de Sitophilus zeamais Motschulsky (Coleoptera: Curculionidae). Boletín Latinoamericano y del Caribe de Plantas Medicinales y Aromáticas 14:48-59.

USEPA. 1993. Probit analysis program. Version 1.5. U.S. Environmental Protection Agency. Washington DC, USA. 